\title{
Risk-Taking Behavior and the Consumption of Alcohol Mixed with Energy Drink among Australian, Dutch and UK Students
}

\author{
Sean J. Johnson ${ }^{1,2}$, Sarah Benson ${ }^{3}\left(\mathbb{D}\right.$, Andrew Scholey $^{3}\left(\mathbb{D}\right.$, Chris Alford $^{1}{ }^{1}$ and Joris C. Verster ${ }^{3,4, *(1)}$ \\ 1 Department of Health and Social Sciences, University of the West of England, Bristol BS16 1QY, UK; \\ JohnsonS11@cardiff.ac.uk (S.J.J.); chris.alford@uwe.ac.uk (C.A.) \\ 2 Centre for Trials Research, Cardiff University, Cardiff CF14 4YS, UK \\ 3 Centre for Human Psychopharmacology, Swinburne University, Melbourne, VIC 3122, Australia; \\ sarahbenson@swin.edu.au (S.B.); andrew@scholeylab.com (A.S.) \\ 4 Division of Pharmacology, Utrecht Institute for Pharmaceutical Sciences (UIPS), Utrecht University, \\ 3584CG Utrecht, The Netherlands \\ * Correspondence: j.c.verster@uu.nl
}

check for

updates

Citation: Johnson, S.J.; Benson, S.; Scholey, A.; Alford, C.; Verster, J.C. Risk-Taking Behavior and the Consumption of Alcohol Mixed with Energy Drink among Australian, Dutch and UK Students. Int. J. Environ. Res. Public Health 2021, 18, 5315. https://doi.org/10.3390/ ijerph18105315

Academic Editor: Paul B. Tchounwou

Received: 18 March 2021

Accepted: 11 May 2021

Published: 17 May 2021

Publisher's Note: MDPI stays neutral with regard to jurisdictional claims in published maps and institutional affiliations.

Copyright: (C) 2021 by the authors. Licensee MDPI, Basel, Switzerland. This article is an open access article distributed under the terms and conditions of the Creative Commons Attribution (CC BY) license (https:// creativecommons.org/licenses/by/ $4.0 /)$.

\begin{abstract}
The relationship between risk-taking behavior, alcohol consumption and negative alcoholrelated consequences is well known. The current analyses were conducted to investigate whether alcohol mixed with energy drink (AMED) is related to risk-taking behavior and if there is a relationship between the amount of energy drink mixed with alcohol consumed, risk-taking behavior and negative alcohol-related consequences. Data from N = 1276 AMED consuming students from the Netherlands, UK and Australia who completed the same survey were evaluated. The analysis revealed that, compared to AMED occasions, on alcohol only (AO) occasions significantly more alcohol was consumed and significantly more negative alcohol-related consequences were reported. On both AO and AMED occasions, there was a strong and positive relationship between amount of alcohol consumed, level of risk-taking behavior and number of reported negative alcohol-related consequences. In contrast, the level of risk-taking behavior was not clearly related to energy drink consumption. Across risk-taking levels, differences in the amount of energy drink consumed on AMED occasions did not exceed one $250 \mathrm{~mL}$ serving of energy drink. When correcting for the amount of alcohol consumed, there were no statistically significant differences in the number of energy drinks consumed on AMED occasions between the risk-taking groups. In conclusion, alcohol consumption is clearly related to risk-taking behavior and experiencing negative alcohol-related consequences. In contrast, energy drink intake was not related to level of risk-taking behavior and only weakly related to the number of experienced negative alcohol-related consequences.
\end{abstract}

Keywords: alcohol; energy drink; alcohol consumption; alcohol-related consequences; risk-taking; students; survey

\section{Introduction}

Excessive alcohol consumption is a persistent, worldwide public health issue. Among 15-49-year olds, alcohol is the leading risk factor for premature mortality and disability, accounting for $10 \%$ of all deaths in this age group [1]. Acute alcohol consumption is partly responsible for this global harm via unintentional injuries, violence and suicide [2]. Consuming too much alcohol in a short space of time has been shown to impact brain functioning, including impaired activity in the pre-frontal cortex that is responsible for executive functions [3]. Acute alcohol consumption has been shown to affect central $\mathrm{GABA}_{\mathrm{A}}$ receptors [4], disrupting the normal processes of the neocortex [5]. The overall effects include greater disinhibitory effects resulting in an increased propensity for risktaking behaviors and alcohol-related harm. Indeed, scientific research has consistently shown that higher levels of alcohol consumption are associated with increased risk-taking behavior and more frequently experiencing negative alcohol-related consequences [6-10]. 
Some risky behaviors associated with alcohol-related consequences include driving under the influence of alcohol [11,12], drinking until blacking out [13], increased violence or aggression [14,15], sexual risk-taking [16] and other risks that may lead to injury [17]. Given the severity of consequences experienced, it is important to examine the factors that might mediate the relationship between heavy alcohol consumption and risk-taking behaviors.

One factor that has been suggested as influencing the relationship between alcohol and risk-taking is energy drinks $[18,19]$. Energy drinks are non-alcoholic beverages, typically containing $80 \mathrm{mg}$ of caffeine per $250 \mathrm{~mL}$ serving size and other functional ingredients such as B-vitamins and taurine. It has been shown that individuals who consume alcohol mixed with energy drink (AMED) have higher risk-taking levels compared to individuals who do not consume AMED and they therefore may consume more alcohol and experience more alcohol-related negative consequences [20-25]. These comparisons do not, however, consider whether both heavier drinking and AMED consumption are a manifestation of some third variable [26]. Indeed, meta-analyses of within-subject comparisons (comparing AMED occasions with alcohol only (AO) occasions within the same subjects) show that the observed increased alcohol consumption is evident in both AMED and AO drinking occasions [26,27]. Similarly, meta-analyses show that individuals who consume AMED do not consume more alcohol on AMED occasions compared to AO occasions [26-28]. In addition, compared to $\mathrm{AO}$ drinking occasions, significantly fewer negative consequences were reported for AMED occasions [23,24].

Notwithstanding these observations, it is of interest to further investigate the possible interplay between energy drink and alcohol consumption and its relationship with risk-taking and experiencing alcohol-related negative consequences $[19,28]$. Previously, Verster et al. [28] concluded that "The literature is overwhelmingly consistent with the notion that AMED consumption is just one manifestation of an underlying trait for greater alcohol consumption along with a cluster of other risky behaviors". Consistent with this literature, we hypothesized that mixing alcohol with energy drinks has no relevant influence on alcohol consumption levels and subsequent negative alcohol-related consequences. We also hypothesized that there would be a direct positive relationship between the amount of alcohol consumed and the level of risk-taking behavior and number of experienced alcohol-related negative consequences, independent of energy drink consumption. The aim of the current study was to further evaluate these hypotheses using large student samples drawn from three countries. Students were recruited as participants because this group comprises regular consumers of AMED [26,28]. The study was conducted in three different countries. This was done in order to verify whether the hypotheses could be confirmed, irrespective of possible different drinking cultures in different countries. Australia, UK and the Netherlands were chosen as in each of these countries both alcohol and energy drink consumption are popular.

\section{Materials and Methods}

Data were evaluated from three directly comparable online surveys conducted among Dutch, UK and Australian students [29-31]. The survey was designed to investigate the possible impact of mixing alcoholic beverages with energy drinks on overall alcohol consumption and alcohol-related consequences. The survey was completed online via SurveyMonkey ${ }^{\circledR}$ (Palo Alto, CA, USA) in Dutch language in the Netherlands and English language in the UK and Australia. The studies were conducted according to the guidelines of the Declaration of Helsinki. The Dutch survey was reviewed by The Medical Ethical Review Board Twente, but no formal medical ethics approval was required. The UK survey was approved by the University of the West of England Faculty Ethics committee (approval number: HAS/14/03/57) and the Australian survey was approved by the Swinburne University Human Research Ethics Committee (Reference 2012/045). Informed consent was obtained from all participants involved in the studies. Detailed information on the content and design of the survey [32] and a comparison between the countries on alcohol intake [33] have been published elsewhere. 
For the current evaluation we investigated the relationship between alcohol and energy drink consumption, risk-taking behavior and negative alcohol-related consequences. These assessments are described in the next sections. Students in the age of 18 to 30 years old who consumed AMED during the past month were included. There were no exclusion criteria.

\subsection{Alcohol and Energy Drink Consumption}

Alcohol consumption questions were adapted from the Quick Drinking Screen $[34,35]$ to assess beverage consumption during the past 30 days. The questions comprised past month's number of alcoholic drinks consumed on a typical ('usual') drinking occasion and on past month's heaviest drinking occasion. Guidance was provided regarding the standardized size of alcoholic drinks using pictures of different serving sizes (e.g., glass, shot, bottle) along with the content in $\mathrm{ml}$, and how to transfer common amounts (e.g., a bottle of wine) into standard units (of $10 \mathrm{~g}$ alcohol in each country). The questions were answered for both AMED and AO drinking occasions. For AMED occasions, it was also assessed how many energy drinks ( 1 unit was defined as a standard serving size of $250 \mathrm{~mL}$ ) were mixed with alcohol on the usual and past month's heaviest drinking occasion. AMED consumption was defined as consuming an energy drink within $+/-2 \mathrm{~h}$ of alcohol consumption, which represents a conservative definition of 'mixing' [32].

\subsection{Level of Risk Taking Behavior}

The risktaking-18 items (RT-18) questionnaire [36] was completed to assess the participants' level of risk-taking behavior. The 18 items can be answered with 'no' or 'yes' ( 0 or 1 point, depending on the item) and the sum score of the RT-18 ranges from 0 (no risktaking) to 18 (extreme risk-taking). Based on previous research [36] and the distribution of the current data, participants were allocated to having a low risk-taking profile (RT-18 score $\leq 5$ ), moderate risk-taking profile (RT-18 score from 6 to 12) or high risk-taking profile (RT-18 score $\geq 13$ ).

\subsection{Negative Alcohol-Related Consequences}

Separate for both AMED and AO drinking occasions, participants completed the Brief Young Adult Alcohol Consequences Questionnaire (BYAACQ) [37,38]. The BYAACQ consists of 24 items representing negative consequences of alcohol consumption. The items could be answered with 'yes' or 'no', depending on whether or not the participant experienced the negative consequence within the past year while drinking AMED and while drinking AO. Based on previous research [38] and the distribution of the current data, participants were allocated to having a low number of negative alcohol-related consequences (BYAACQ score $\leq 4$ ), having a moderate number of negative alcohol-related consequences (BYAACQ score of 5-8) or having a high number of negative alcohol-related consequences (BYAACQ score $\geq 9$ ).

\subsection{Statistical Analysis}

Data were analyzed using IBM SPSS Version 27 (IBM Corp., Armonk, NY, USA). For the current analysis, participants were included if they were students between the age of 18 and 30 years old and consumed AMED during the past month. Participants with missing data were excluded from the analysis. Moreover, data on energy drink consumption from three UK participants that reported unreliably high numbers of energy drinks on their heaviest drinking occasion ( $\geq 30250 \mathrm{~mL}$ energy drinks) were also omitted from the analysis. The mean and standard deviation (SD) were computed for all variables and the distribution of the data was checked for normality. Alcohol and energy drink consumption was compared between the levels of risk-taking behavior and between the levels of experienced negative alcohol-related consequences. These comparisons were performed for the sample as a whole and for the individual countries. As the data were not normally distributed, the Independent-Samples Kruskal-Wallis test was used. If the main effect was statistically significant, post-hoc pairwise comparisons were conducted, applying 
appropriate Bonferroni correction to account for multiple comparisons. Differences were considered statistically significant if $p<0.05$. Sex differences were evaluated with the Independent-Samples Mann-Whitney $U$ test. Differences between men and women were considered statistically significant if $p<0.05$.

\section{Results}

Of the $\mathrm{N}=2205$ AMED consumers who started the survey, $\mathrm{N}=1267$ completed all assessments. The sample under evaluation comprised 1267 AMED consumers $(\mathrm{N}=553$ men and $\mathrm{N}=714$ women). Their demographics are summarized in Table 1.

Table 1. Demographics and study outcomes.

\begin{tabular}{ccccc}
\hline Demographics & Overall & The Netherlands & UK & Australia \\
\hline $\mathrm{N}$ & 1267 & 713 & 441 & 113 \\
Male/female ratio & $553 / 714$ & $306 / 407$ & $209 / 232$ & $38 / 75$ \\
Age (years) & $21.1(2.3)$ & $21.4(2.3)$ & $20.5(2.0) \Upsilon$ & $20.9(2.7)^{\dagger}$ \\
Age of regular alcohol use & $16.7(1.7)$ & $16.5(1.7)$ & $16.9(1.7) \Upsilon$ & $17.1(1.9)^{\dagger}$ \\
RT-18 total risk-taking score & $8.3(4.1)$ & $7.5(4.1)$ & $9.0(4.1) \Upsilon$ & $10.4(3.3)^{\dagger \ddagger}$ \\
BYAACQ AO & $7.2(5.0)$ & $5.4(4.0)$ & $9.5(5.4) \Upsilon$ & $9.1(4.6)^{\dagger}$ \\
BYAACQ AMED & $4.9(4.8)$ & $3.1(3.6)$ & $7.6(5.1) \Upsilon$ & $6.5(4.3)^{\dagger}$ \\
\hline
\end{tabular}

Mean and standard deviation (SD, between brackets) are shown. Significant differences, after Bonferroni's correction for multiple comparisons, between the countries are indicated as follows: The Netherlands and UK by $\checkmark$, between the Netherlands and Australia by ${ }^{\dagger}$ and between UK and Australia by ${ }^{\ddagger}$. Abbreviations: RT- $18=$ risktaking questionnaire, 18 items; $\mathrm{BYAACQ}=$ brief young adult alcohol consequences questionnaire; $\mathrm{AO}=\mathrm{alcohol}$ only; AMED = alcohol mixed with energy drink.

In each country, significantly more women than men completed the survey $(p<0.0001$ for Netherlands and Australia, $p<0.02$ for UK). Dutch participants were significantly younger $(p<0.0001)$ and started consuming alcohol regularly at a younger age compared to UK $(p<0.0001)$ and Australian students $(p<0.0001)$. Risk-taking scores and reported negative alcohol-related consequences were significantly lower in the Netherlands compared to the UK and Australian sample ( $p<0.0001$ for each pairwise comparison).

Figure 1 illustrates the relationship between risk-taking and alcohol and energy drink consumption. The trendlines in Figure 1 suggest that for both usual and the past month's heaviest $\mathrm{AO}$ and $\mathrm{AMED}$ occasions there is a clear relationship between alcohol intake and level of risk-taking behavior, whereas this relationship is not seen between risk-taking behavior and the number of energy drinks consumed on AMED occasions.

Table 2 summarized alcohol intake according to risk-taking behavior level. Table 3 summarized the number of energy drinks consumed on AMED occasions according to risk-taking behavior level.

Table 2 summarizes the number of alcoholic drinks consumed on AMED and AO occasions (usual and past month heaviest drinking occasion) for the three countries and the three risk-taking levels. Within-subject comparisons revealed that, compared to AMED occasions, participants consumed significantly more alcohol on both usual AO occasions $(p<0.0001)$ and past month heaviest AO drinking occasions $(p<0.0001)$. This observation was consistent for usual drinking occasions across the three countries $(p<0.0001$ for the Netherlands and UK; $p=0.001$ for Australia) and across risk-taking groups (all comparisons $p<0.0001)$. The observation was also consistent for past month heaviest drinking occasions across the three countries (all comparisons $p<0.0001$ ) and across risk-taking groups (all comparisons $p<0.0001)$.

It is also evident from Table 2 that with higher levels of risk-taking the reported number of alcoholic drinks consumed was higher, both on AO and AMED occasions, and both on usual drinking occasions and past month heaviest drinking occasion. Table 3 shows that the differences in number of energy drinks consumed on AMED occasions between the risk-taking groups are small, i.e., $\leq 1$ serving of $250 \mathrm{~mL}$ energy drink. When correcting 
for the number of alcoholic drinks consumed, the number of energy drinks consumed on AMED occasions did not significantly differ between any of the risk-taking groups.
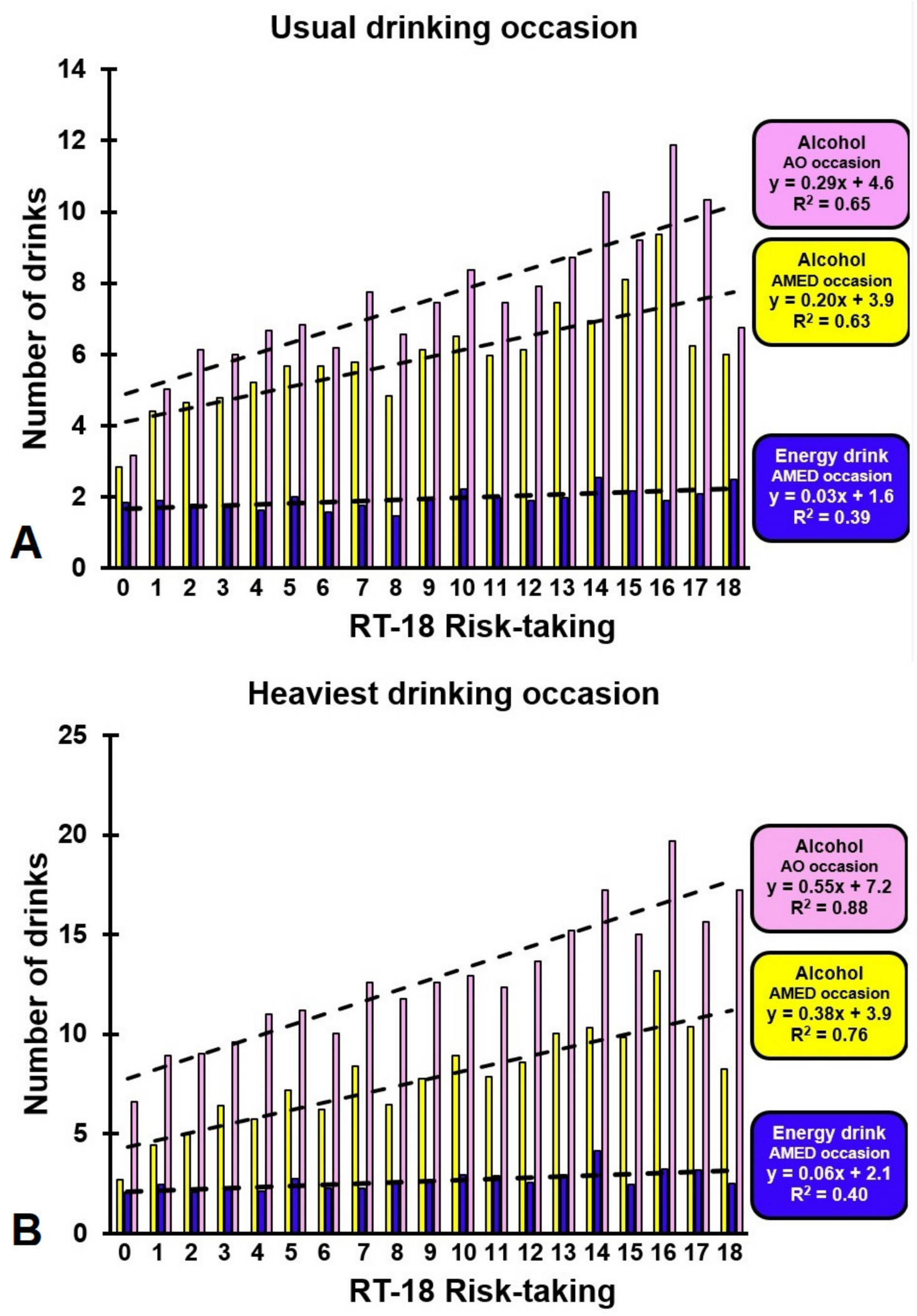

Figure 1. The relationship between risk-taking and alcohol and energy drink consumption. Shown are the mean number of drinks consumed for each RT-18 risk-taking score for usual drinking occasions (A) and past month heaviest drinking occasion (B). Abbreviations: $\mathrm{AO}=$ alcohol only; $\mathrm{AMED}=$ alcohol mixed with energy drink; RT-18 = risk-taking questionnaire, 18 items. 
Table 2. Alcohol consumption and risk-taking.

\begin{tabular}{|c|c|c|c|c|c|c|c|}
\hline \multirow[b]{2}{*}{$\begin{array}{c}\text { RT-18 Risk } \\
\text { Taking Group }\end{array}$} & \multicolumn{3}{|c|}{ Number of Alcoholic Drinks } & \multirow{2}{*}{$\begin{array}{l}\text { Overall } \\
p \text {-Value }\end{array}$} & \multicolumn{3}{|c|}{ Pairwise Comparisons } \\
\hline & $\begin{array}{l}\text { Low } \\
(0-5)\end{array}$ & $\begin{array}{l}\text { Moderate } \\
(6-12)\end{array}$ & $\begin{array}{c}\text { High } \\
\text { (13-18) }\end{array}$ & & $\begin{array}{l}\text { Low vs. } \\
\text { Moderate }\end{array}$ & $\begin{array}{c}\text { Moderate vs. } \\
\text { High }\end{array}$ & $\begin{array}{l}\text { Low vs. } \\
\text { High }\end{array}$ \\
\hline \multicolumn{8}{|c|}{ Usual AO occasion } \\
\hline Overall & $6.1(4.5)$ & $7.4(4.8)$ & $9.8(6.1)$ & $<0.0001 *$ & $<0.0001 *$ & $<0.0001^{*}$ & $<0.0001^{*}$ \\
\hline The Netherlands & $5.3(3.8)$ & $6.6(3.9)$ & $8.1(4.5)$ & $<0.0001 *$ & $<0.0001 *$ & $<0.0001 *$ & $0.002 *$ \\
\hline UK & $8.3(5.6)$ & $9.2(5.7)$ & $12.3(7.2)$ & $<0.0001 *$ & 0.215 & $0.001 *$ & $<0.0001$ * \\
\hline Australia & $4.8(1.9)$ & $5.8(3.2)$ & $7.6(4.1)$ & 0.053 & - & - & - \\
\hline \multicolumn{8}{|c|}{ Past month heaviest AO occasion } \\
\hline Overall & $10.1(6.3)$ & $12.3(6.9)$ & $16.4(8.7)$ & $<0.0001 *$ & $<0.0001 *$ & $<0.0001^{*}$ & $<0.0001 *$ \\
\hline The Netherlands & $9.5(5.6)$ & $11.9(6.2)$ & $15.6(7.9)$ & $<0.0001 *$ & $<0.0001 *$ & $<0.0001 *$ & $<0.0001 *$ \\
\hline UK & $11.9(7.5)$ & $13.9(8.0)$ & $18.1(9.3)$ & $<0.0001 *$ & 0.078 & $<0.0001 *$ & $<0.0001$ * \\
\hline Australia & $6.5(3.7)$ & $9.2(4.5)$ & $13.3(8.0)$ & $0.007 *$ & 0.354 & $0.045 *$ & $0.016 *$ \\
\hline \multicolumn{8}{|c|}{ Usual AMED occasion } \\
\hline Overall & $5.0(3.4)$ & $5.9(3.9)$ & $7.6(4.8)$ & $<0.0001 *$ & $<0.0001 *$ & $<0.0001 *$ & $<0.0001 *$ \\
\hline The Netherlands & $4.8(3.1)$ & $6.0(3.9)$ & $7.7(4.5)$ & $<0.0001 *$ & $0.001 *$ & $0.001 *$ & $<0.0001$ * \\
\hline UK & $5.5(4.2)$ & $6.0(4.3)$ & $8.0(5.2)$ & $0.001 *$ & 0.839 & $0.005 *$ & $0.001 *$ \\
\hline Australia & $3.8(2.0)$ & $4.9(2.9)$ & $6.2(4.0)$ & 0.166 & - & - & - \\
\hline \multicolumn{8}{|c|}{ Past month heaviest AMED occasion } \\
\hline Overall & $5.8(5.1)$ & $7.8(6.2)$ & $10.5(8.6)$ & $<0.0001 *$ & $<0.0001^{*}$ & $<0.0001 *$ & $<0.0001 *$ \\
\hline The Netherlands & $5.2(4.2)$ & $7.4(5.8)$ & $9.4(7.8)$ & $<0.0001 *$ & $<0.0001 *$ & 0.127 & $<0.0001$ * \\
\hline UK & $7.2(6.9)$ & $8.6(7.1)$ & $11.7(8.9)$ & $<0.0001 *$ & 0.132 & $0.003 *$ & $<0.0001^{*}$ \\
\hline Australia & $5.8(3.4)$ & $6.9(4.7)$ & $10.6(9.8)$ & 0.378 & - & - & - \\
\hline
\end{tabular}

Mean and standard deviation (SD, between brackets) number of alcoholic drinks are shown. Post-hoc pairwise comparisons were conducted with Bonferroni's correction. ${ }^{*}=$ Differences are significant if $p<0.05 .-=$ no post-hoc tests conducted as main effect was not significant. Abbreviations: $\mathrm{AO}=$ alcohol only; $\mathrm{AMED}=$ alcohol mixed with energy drink.

Table 3. Energy drinks mixed with alcohol consumption and risk-taking.

\begin{tabular}{|c|c|c|c|c|c|}
\hline \multirow[b]{2}{*}{$\begin{array}{l}\text { RT-18 Risk Taking } \\
\text { Group }\end{array}$} & \multicolumn{3}{|c|}{ Number of Energy Drinks on AMED Occasion } & \multicolumn{2}{|l|}{ Overall } \\
\hline & $\begin{array}{l}\text { Low } \\
(0-5)\end{array}$ & $\begin{array}{l}\text { Moderate } \\
(6-12)\end{array}$ & $\begin{array}{c}\text { High } \\
\text { (13-18) }\end{array}$ & $p$-Value & Corrected $p$-Value ${ }^{1}$ \\
\hline \multicolumn{6}{|c|}{ Usual AMED Occasion } \\
\hline Overall & $1.8(1.3)$ & $1.8(1.5)$ & $2.2(1.9)$ & $0.011 *$ & 0.457 \\
\hline The Netherlands & $1.7(1.3)$ & $1.6(1.0)$ & $1.9(1.8)$ & 0.464 & 0.073 \\
\hline UK & $2.1(1.3)$ & $2.3(2.0)$ & $2.6(2.2)$ & 0.219 & 0.744 \\
\hline Australia & $2.1(1.4)$ & $1.7(1.4)$ & $1.7(0.8)$ & 0.171 & 0.271 \\
\hline \multicolumn{6}{|c|}{ Past Month Heaviest AMED Occasion } \\
\hline Overall & $2.3(1.5)$ & $2.6(2.2)$ & $3.2(2.8)$ & $0.001 *$ & 0.053 \\
\hline The Netherlands & $2.2(1.5)$ & $2.3(2.0)$ & $3.0(2.9)$ & 0.118 & 0.069 \\
\hline UK & $2.7(1.6)$ & $3.0(2.2)$ & $3.7(3.0)$ & $0.016^{*}$ & 0.128 \\
\hline Australia & $2.0(0.8)$ & $2.5(2.6)$ & $2.1(1.1)$ & 0.798 & 0.315 \\
\hline
\end{tabular}

Mean and standard deviation (SD, between brackets) number of energy drinks consumed on AMED occasions are shown. Post-hoc pairwise comparisons were conducted with Bonferroni's correction. ${ }^{*}=$ Differences are significant if $p<0.05 .{ }^{1}=p$-value corrected for the number of alcoholic drinks consumed. Abbreviations: AMED = alcohol mixed with energy drink.

Table 4 summarizes alcohol consumption for the three BYAACQ groups. Table 4 shows that, consistent across countries and observed for both AO and AMED occasions, increasing numbers of negative alcohol-related consequences are associated with higher levels of alcohol intake.

Table 5 summarized the relationship between the number of energy drinks consumed on AMED occasions and reported negative alcohol-related consequences. The analysis revealed that participants who reported more negative alcohol-related consequences con- 
sumed more energy drinks on AMED occasions. However, the absolute differences in number of energy drinks consumed between the groups was small and except for the UK equaled less than one $250 \mathrm{~mL}$ serving of energy drink between the lowest and highest BYAACQ groups. Of interest, energy drink intake of the moderate BYAACQ group of the Dutch and Australian sample was higher compared to the highest BYAACQ group.

Table 4. Alcohol consumption and negative alcohol-related consequences.

\begin{tabular}{|c|c|c|c|c|c|c|c|}
\hline \multirow[b]{2}{*}{ BYAACQ Group } & \multicolumn{3}{|c|}{ Number of Alcoholic Drinks } & \multirow{2}{*}{$\begin{array}{l}\text { Overall } \\
p \text {-Value }\end{array}$} & \multicolumn{3}{|c|}{ Pairwise Comparisons } \\
\hline & $\begin{array}{l}\text { Low } \\
(0-4)\end{array}$ & $\begin{array}{c}\text { Moderate } \\
(5-8)\end{array}$ & $\begin{array}{l}\text { High } \\
(9-24)\end{array}$ & & $\begin{array}{l}\text { Low vs. } \\
\text { Moderate }\end{array}$ & $\begin{array}{l}\text { Moderate vs. } \\
\text { High }\end{array}$ & $\begin{array}{l}\text { Low vs. } \\
\text { High }\end{array}$ \\
\hline \multicolumn{8}{|c|}{ Usual AO Occasion } \\
\hline Overall & $5.4(3.5)$ & $7.1(4.5)$ & $9.9(5.9)$ & $<0.0001 *$ & $<0.0001 *$ & $<0.0001 *$ & $<0.0001 *$ \\
\hline The Netherlands & $5.0(3.2)$ & $6.8(4.3)$ & $9.0(4.3)$ & $<0.0001 *$ & $<0.0001 *$ & $<0.0001 *$ & $<0.0001 *$ \\
\hline UK & $6.9(4.5)$ & $8.5(4.8)$ & $11.2(6.8)$ & $<0.0001 *$ & $0.042 *$ & $0.001 *$ & $<0.0001$ * \\
\hline Australia & $5.1(2.5)$ & $5.2(3.6)$ & $7.1(3.3)$ & $0.001 *$ & 1.000 & 0.001 * & 0.060 \\
\hline \multicolumn{8}{|c|}{ Past Month Heaviest AO Occasion } \\
\hline Overall & $9.0(5.4)$ & $12.3(6.6)$ & $16.0(8.0)$ & $<0.0001 *$ & $<0.0001 *$ & $<0.0001 *$ & $<0.0001 *$ \\
\hline The Netherlands & $8.8(5.1)$ & $12.5(5.7)$ & $17.0(7.2)$ & $<0.0001 *$ & $<0.0001 *$ & $<0.0001 *$ & $<0.0001$ * \\
\hline UK & $10.2(6.5)$ & $13.4(8.1)$ & $16.2(8.6)$ & $<0.0001 *$ & 0.018 * & $0.006^{*}$ & $<0.0001$ * \\
\hline Australia & $7.0(3.9)$ & $8.2(4.4)$ & $12.5(6.4)$ & $<0.0001 *$ & 1.000 & $<0.0001 *$ & $0.001 *$ \\
\hline \multicolumn{8}{|c|}{ Usual AMED Occasion } \\
\hline Overall & $4.9(3.3)$ & $7.1(4.5)$ & $7.4(4.6)$ & $<0.0001 *$ & $<0.0001 *$ & $<0.0001 *$ & $<0.0001$ * \\
\hline The Netherlands & $5.1(3.3)$ & $8.5(4.6)$ & $7.7(4.2)$ & $<0.0001 *$ & $<0.0001 *$ & 1.000 & $<0.0001 *$ \\
\hline UK & $4.7(3.7)$ & $6.5(4.5)$ & $7.5(4.9)$ & $<0.0001 *$ & $0.002 *$ & 0.227 & $<0.0001 *$ \\
\hline Australia & $4.2(2.5)$ & $5.3(2.7)$ & $6.3(4.0)$ & $0.024^{*}$ & 0.222 & 1.000 & $0.028 *$ \\
\hline \multicolumn{8}{|c|}{ Past Month Heaviest AMED Occasion } \\
\hline Overall & $6.1(5.2)$ & $10.0(8.0)$ & $10.0(7.3)$ & $<0.0001 *$ & $<0.0001 *$ & $<0.0001 *$ & $<0.0001 *$ \\
\hline The Netherlands & $5.8(4.8)$ & $10.5(6.9)$ & $11.0(7.6)$ & $<0.0001 *$ & $<0.0001 *$ & 1.000 & $<0.0001 *$ \\
\hline UK & $6.8(6.3)$ & $10.4(9.3)$ & $9.7(6.9)$ & $<0.0001 *$ & $<0.0001 *$ & 1.000 & $<0.0001 *$ \\
\hline Australia & $6.6(5.4)$ & $7.1(4.6)$ & $9.9(8.7)$ & 0.255 & - & - & - \\
\hline
\end{tabular}

Mean and SD (between brackets) number of alcoholic drinks are shown. Post-hoc pairwise comparisons were conducted with Bonferroni's correction. ${ }^{*}=$ Differences are significant if $p<0.05 .-=$ no post-hoc tests conducted as main effect was not significant. Abbreviations: $\mathrm{BYAACQ}=$ brief young adult alcohol consequences questionnaire; $\mathrm{AO}=$ alcohol only; $\mathrm{AMED}=$ alcohol mixed with energy drink.

Table 5. Energy drinks mixed with alcohol consumption and negative consequences.

\begin{tabular}{|c|c|c|c|c|c|c|c|c|}
\hline \multirow[b]{2}{*}{$\begin{array}{l}\text { BYAACQ } \\
\text { Group }\end{array}$} & \multicolumn{3}{|c|}{$\begin{array}{c}\text { Number of Energy Drinks on } \\
\text { AMED Occasion }\end{array}$} & \multirow{2}{*}{$\begin{array}{l}\text { Overall } \\
p \text {-Value }\end{array}$} & \multicolumn{3}{|c|}{ Pairwise Comparisons } & \multirow[b]{2}{*}{$\begin{array}{l}\text { Corrected } \\
p \text {-Value }\end{array}$} \\
\hline & $\begin{array}{l}\text { Low } \\
(0-4)\end{array}$ & $\begin{array}{l}\text { Moderate } \\
\quad(5-8)\end{array}$ & $\begin{array}{l}\text { High } \\
(9-24)\end{array}$ & & $\begin{array}{l}\text { Low vs. } \\
\text { Moderate }\end{array}$ & $\begin{array}{l}\text { Moderate } \\
\text { vs. High }\end{array}$ & $\begin{array}{l}\text { Low vs. } \\
\text { High }\end{array}$ & \\
\hline \multicolumn{9}{|c|}{ Usual AMED Occasion } \\
\hline Overall & $1.6(1.2)$ & $2.1(1.8)$ & $2.4(1.9)$ & $<0.0001 *$ & $<0.0001 *$ & $0.012 *$ & $<0.0001 *$ & $<0.0001$ * \\
\hline The Netherlands & $1.6(1.1)$ & $2.0(1.7)$ & $1.6(1.0)$ & 0.119 & - & - & - & $0.027 *$ \\
\hline UK & $1.8(1.2)$ & $2.2(2.0)$ & $2.8(2.2)$ & $<0.0001 *$ & 0.459 & $0.001 *$ & $<0.0001 *$ & $0.014 *$ \\
\hline Australia & $1.6(1.2)$ & $2.0(1.8)$ & $1.6(0.9)$ & 0.239 & - & - & - & 0.135 \\
\hline \multicolumn{9}{|c|}{ Past Month Heaviest AMED Occasion } \\
\hline Overall & $2.2(1.8)$ & $3.0(2.5)$ & $3.3(2.5)$ & $<0.0001 *$ & $<0.0001^{*}$ & 0.347 & $<0.0001 *$ & $<0.0001^{*}$ \\
\hline The Netherlands & $2.2(1.8)$ & $3.1(2.9)$ & $2.7(1.8)$ & $<0.0001 *$ & $<0.0001 *$ & 1.000 & $0.018^{*}$ & 0.093 \\
\hline UK & $2.4(1.8)$ & $3.0(1.9)$ & $3.7(2.7)$ & $<0.0001$ * & $0.004 *$ & 0.120 & $<0.0001^{*}$ & $<0.0001 *$ \\
\hline Australia & $2.0(1.6)$ & $2.8(3.2)$ & $2.4(1.9)$ & 0.239 & - & - & - & 0.365 \\
\hline
\end{tabular}

Mean and SD (between brackets) number of energy drinks consumed on AMED occasions are shown. Post-hoc pairwise comparisons were conducted with Bonferroni's correction. - = no post-hoc tests conducted as main effect was not significant. * $=$ Differences are significant if $p<0.05 .{ }^{1}=p$-value corrected for the number of alcoholic drinks consumed. Abbreviations: BYAACQ = brief young adult alcohol consequences questionnaire; $\mathrm{AMED}=$ alcohol mixed with energy drink. 


\section{Sex Differences}

To evaluate possible sex differences we analyzed the combined dataset, comparing outcomes of 553 male and 714 female participants. Women started consuming alcohol regularly at a later age then men and consume significantly less alcohol (both quantity and frequency) on AMED and AO occasions compared to men (See Table 6). The data show that on both AO and AMED occasions women consumed significantly less alcohol than men. Women also consumed significantly fewer energy drinks on AMED occasions. Further, women had significantly lower risk-taking scores and reported significantly fewer negative alcohol-related consequences for both AO and AMED occasions. No sex differences were found for age or age of regular alcohol consumption.

Table 6. Sex differences.

\begin{tabular}{cccc}
\hline Demographics and Assessments & $\begin{array}{c}\text { Men } \\
(\mathbf{N}=\mathbf{5 5 3})\end{array}$ & $\begin{array}{c}\text { Women } \\
\mathbf{( N = 7 1 4 )}\end{array}$ & $p$-Value \\
\hline Age (years) & $21.0(2.3)$ & $21.1(2.3)$ & 0.941 \\
Age of regular alcohol use & $16.6(1.7)$ & $16.8(1.7)$ & 0.247 \\
RT-18 total risk-taking score & $9.1(4.0)$ & $7.7(4.2)$ & $<0.0001^{*}$ \\
\hline AO occasions & & & \\
\hline Number of alcoholic drinks (usual occasion) & $9.3(5.9)$ & $6.0(3.8)$ & $<0.0001^{*}$ \\
Number of alcoholic drinks (heaviest occasion) & $15.6(8.0)$ & $9.9(5.6)$ & $<0.0001^{*}$ \\
BYAACQ (AO occasions) & $8.3(5.2)$ & $6.4(4.7)$ & $<0.0001^{*}$ \\
\hline AMED occasions & & & \\
Number of alcoholic drinks (usual occasion) & $7.3(4.7)$ & $4.8(3.0)$ & $<0.0001^{*}$ \\
Number of energy drinks (usual occasion) & $2.1(1.8)$ & $1.7(1.2)$ & $<0.0001^{*}$ \\
Number of alcoholic drinks (heaviest occasion) & $9.8(7.6)$ & $6.1(5.2)$ & $<0.0001^{*}$ \\
Number of energy drinks (heaviest occasion) & $3.1(2.6)$ & $2.3(1.7)$ & $<0.0001^{*}$ \\
BYAACQ (AMED occasions) & $5.9(5.2)$ & $4.2(4.3)$ & $<0.0001^{*}$ \\
\hline
\end{tabular}

Mean and standard deviation (SD, between brackets) are shown. Significant differences $(p<0.05)$ between men and women are indicated by *. Abbreviations: BYAACQ = brief young adult alcohol consequences questionnaire; $\mathrm{AO}=$ alcohol only; $\mathrm{AMED}=$ alcohol mixed with energy drink .

Table 7 summarizes alcohol consumption for the three risk-taking groups for men and women. Among both men and women, the number of alcoholic drinks was significantly higher at subsequent levels of risk-taking. For each comparison at each risk-taking level, men consumed significantly more alcohol than women $(p<0.05)$. Table 8 summarizes energy drink consumption for the three risk-taking groups for men and women. While energy drink intake increased at higher risk-taking levels for both men and women, except for the difference in energy drink consumption between the lowest and highest risk-taking level of men on their heaviest drinking occasion (1.3 servings, $p=0.010)$, the magnitude of the all other differences in energy drink consumption between the risk-taking levels was always less than one can of energy drink and not statistically significant. Taken together, among both men and women a clear relationship between the level of risk-taking and alcohol consumption was observed, whereas the differences in energy drink consumption were usually not significant between the risk-taking levels.

Table 7. Alcohol consumption and risk-taking.

\begin{tabular}{cccccccc}
\hline & \multicolumn{2}{c}{ Number of Alcoholic Drinks } & Overall & \multicolumn{2}{c}{ Pairwise Comparisons } \\
\hline $\begin{array}{c}\text { RT-18 Risk Taking } \\
\text { Group }\end{array}$ & $\begin{array}{c}\text { Low } \\
\mathbf{( 0 - 5 )}\end{array}$ & $\begin{array}{c}\text { Moderate } \\
\mathbf{( 6 - 1 2 )}\end{array}$ & $\begin{array}{c}\text { High } \\
\mathbf{( 1 3 - 1 8 )}\end{array}$ & $\boldsymbol{p}$-Value & $\begin{array}{c}\text { Low vs. } \\
\text { Moderate }\end{array}$ & $\begin{array}{c}\text { Moderate vs. } \\
\text { High }\end{array}$ & $\begin{array}{c}\text { Low vs. } \\
\text { High }\end{array}$ \\
\hline & \multicolumn{9}{c}{ Usual AO Occasion } \\
\hline Men & $7.6(5.7)$ & $9.0(5.3)$ & $11.6(7.0)$ & $<0.0001^{*}$ & $0.003^{*}$ & $0.001^{*}$ & $<0.0001^{*}$ \\
Women & $5.4(3.6)$ & $6.0(3.7)$ & $7.6(3.9)$ & $<0.0001^{*}$ & $0.017^{*}$ & $<0.0001^{*}<0.0001^{*}$ \\
\hline
\end{tabular}


Table 7. Cont.

\begin{tabular}{|c|c|c|c|c|c|c|c|}
\hline \multirow[b]{2}{*}{$\begin{array}{l}\text { RT-18 Risk Taking } \\
\text { Group }\end{array}$} & \multicolumn{3}{|c|}{ Number of Alcoholic Drinks } & \multirow{2}{*}{$\begin{array}{l}\text { Overall } \\
p \text {-Value }\end{array}$} & \multicolumn{3}{|c|}{ Pairwise Comparisons } \\
\hline & $\begin{array}{l}\text { Low } \\
(0-5)\end{array}$ & $\begin{array}{c}\text { Moderate } \\
(6-12)\end{array}$ & $\begin{array}{c}\text { High } \\
\text { (13-18) }\end{array}$ & & $\begin{array}{l}\text { Low vs. } \\
\text { Moderate }\end{array}$ & $\begin{array}{l}\text { Moderate vs. } \\
\text { High }\end{array}$ & $\begin{array}{l}\text { Low vs. } \\
\text { High }\end{array}$ \\
\hline \multicolumn{8}{|c|}{ Past month heaviest AO Occasion } \\
\hline Men & $13.0(7.1)$ & $15.3(7.5)$ & $18.8(9.2)$ & $<0.0001 *$ & $0.009 *$ & $0.002 *$ & $<0.0001 *$ \\
\hline Women & $8.5(5.3)$ & $9.8(5.1)$ & $13.4(6.9)$ & $<0.0001 *$ & $0.003 *$ & $<0.0001 *$ & $<0.0001 *$ \\
\hline \multicolumn{8}{|c|}{ Usual AMED Occasion } \\
\hline Men & $6.5(4.2)$ & $7.2(4.5)$ & $8.5(5.5)$ & $0.014 *$ & 0.416 & 0.125 & $0.011 *$ \\
\hline Women & $4.2(2.7)$ & $4.7(2.9)$ & $6.5(3.5)$ & $<0.0001 *$ & 0.119 & $<0.0001 *$ & $<0.0001 *$ \\
\hline \multicolumn{8}{|c|}{ Past month heaviest AMED Occasion } \\
\hline Men & $7.1(6.1)$ & $9.7(6.8)$ & $12.5(9.7)$ & $<0.0001 *$ & $<0.0001 *$ & 0.111 & $<0.0001 *$ \\
\hline Women & $5.2(4.4)$ & $6.2(5.2)$ & $8.1(6.3)$ & $<0.0001 *$ & 0.044 * & $0.002 *$ & $<0.0001$ * \\
\hline
\end{tabular}

Mean and SD (between brackets) number of alcoholic drinks are shown. Post-hoc pairwise comparisons were conducted with Bonferroni's correction. ${ }^{*}=$ Differences are significant if $p<0.05$. Abbreviations: $\mathrm{AO}=$ alcohol only; $\mathrm{AMED}=$ alcohol mixed with energy drink.

Table 8. Energy drinks mixed with alcohol consumption and risk-taking.

\begin{tabular}{|c|c|c|c|c|c|}
\hline \multirow[b]{2}{*}{ RT-18 Risk Taking Group } & \multicolumn{3}{|c|}{ Number of Energy Drinks on AMED Occasion } & \multicolumn{2}{|l|}{ Overall } \\
\hline & $\begin{array}{l}\text { Low } \\
(0-5)\end{array}$ & $\begin{array}{c}\text { Moderate } \\
(6-12)\end{array}$ & $\begin{array}{c}\text { High } \\
(13-18)\end{array}$ & $p$-Value & Corrected $p$-Value ${ }^{1}$ \\
\hline \multicolumn{6}{|c|}{ Usual AMED occasion } \\
\hline Men & $1.8(1.3)$ & $2.1(1.8)$ & $2.4(2.3)$ & $0.049 *$ & 0.198 \\
\hline Women & $1.8(1.3)$ & $1.6(1.2)$ & $1.9(1.3)$ & 0.046 * & 0.077 \\
\hline \multicolumn{6}{|c|}{ Past Month Heaviest AMED Occasion } \\
\hline Men & $2.4(1.6)$ & $3.1(2.6)$ & $3.7(3.2)$ & $0.001 *$ & $0.036^{*}$ \\
\hline Women & $2.3(1.5)$ & $2.2(1.6)$ & $2.6(2.2)$ & $0.025 *$ & 0.148 \\
\hline
\end{tabular}

Mean and SD (between brackets) number of energy drinks consumed on AMED occasions are shown. Post-hoc pairwise comparisons were conducted with Bonferroni's correction. ${ }^{*}=$ Differences are significant if $p<0.05 .{ }^{1}=p$-value corrected for the number of alcoholic drinks consumed. Abbreviations: AMED = alcohol mixed with energy drink.

Table 9 summarizes alcohol consumption for the three BYAACQ groups for men and women. Among both men and women, the number of alcoholic drinks was significantly higher at subsequent BYAACQ levels. For each comparison at each risk-taking level, men consumed significantly more alcohol than women $(p<0.05)$. Table 10 summarizes energy drink consumption for the BYAACQ groups for men and women. While energy drink intake increased at higher BYAACQ levels for both men and women, the absolute differences were small and less than one can of energy drink. For usual AMED occasions, the difference in energy drink consumption between the lowest and highest BYAACQ level was significant in both men $(0.9$ servings, $p<0.0001)$ and women $(0.5$ servings, $p=0.023)$ and in men only between the medium and highest BYAAC level ( 0.6 servings, $p=0.002)$ were statistically significant. For the heaviest drinking occasion, post-hoc analysis revealed significant differences between energy drink consumption between the lowest and highest BYAAC level for both men (1.2 servings, $p<0.0001)$ and women $(0.6$ serving, $p=0.016)$. All other differences in energy drink consumption between the BYAACQ levels were not statistically significant. Taken together, among both men and women a clear relationship between the level of experienced negative alcohol-related consequences and alcohol consumption were observed. In contrast, differences in energy drink consumption between the BYAACQ levels were usually less than one can of energy drink. 
Table 9. Alcohol consumption and negative alcohol-related consequences.

\begin{tabular}{|c|c|c|c|c|c|c|c|}
\hline \multirow[b]{2}{*}{ BYAACQ Group } & \multicolumn{3}{|c|}{ Number of Alcoholic Drinks } & \multirow{2}{*}{$\begin{array}{l}\text { Overall } \\
p \text {-Value }\end{array}$} & \multicolumn{3}{|c|}{ Pairwise Comparisons } \\
\hline & $\begin{array}{l}\text { Low } \\
(0-4)\end{array}$ & $\begin{array}{l}\text { Moderate } \\
\quad(5-8)\end{array}$ & $\begin{array}{l}\text { High } \\
(9-24)\end{array}$ & & $\begin{array}{l}\text { Low vs. } \\
\text { Moderate }\end{array}$ & $\begin{array}{c}\text { Moderate vs. } \\
\text { High }\end{array}$ & $\begin{array}{l}\text { Low vs. } \\
\text { High }\end{array}$ \\
\hline \multicolumn{8}{|c|}{ Usual AO Occasion } \\
\hline Men & $6.9(4.4)$ & $8.4(5.3)$ & $11.5(6.4)$ & $<0.0001^{*}$ & 0.011 * & $<0.0001 *$ & $<0.0001 *$ \\
\hline Women & $4.6(2.7)$ & $6.1(3.4)$ & $8.0(4.5)$ & $<0.0001^{*}$ & $<0.0001 *$ & $<0.0001 *$ & $<0.0001$ * \\
\hline \multicolumn{8}{|c|}{ Past Month Heaviest AO Occasion } \\
\hline Men & $11.4(6.2)$ & $14.9(7.3)$ & $18.7(8.4)$ & $<0.0001 *$ & $<0.0001 *$ & $<0.0001 *$ & $<0.0001$ * \\
\hline Women & $7.6(4.5)$ & $10.3(5.2)$ & $12.7(6.3)$ & $<0.0001 *$ & $<0.0001 *$ & $0.001 *$ & $<0.0001$ * \\
\hline \multicolumn{8}{|c|}{ Usual AMED Occasion } \\
\hline Men & $6.1(4.1)$ & $8.8(5.0)$ & $8.3(5.0)$ & $<0.0001^{*}$ & $<0.0001 *$ & 1.000 & $<0.0001 *$ \\
\hline Women & $4.2(2.6)$ & $5.5(3.3)$ & $6.2(3.7)$ & $<0.0001 *$ & $<0.0001 *$ & 0.640 & $<0.0001$ * \\
\hline \multicolumn{8}{|c|}{ Past Month Heaviest AMED Occasion } \\
\hline Men & $7.7(6.2)$ & $12.4(8.8)$ & $11.3(7.8)$ & $<0.0001 *$ & $<0.0001 *$ & 1.000 & $<0.0001 *$ \\
\hline Women & $5.1(4.1)$ & $7.8(6.4)$ & $8.2(6.2)$ & $<0.0001^{*}$ & $<0.0001 *$ & 1.000 & $<0.0001 *$ \\
\hline
\end{tabular}

Mean and SD (between brackets) number of alcoholic drinks are shown. Post-hoc pairwise comparisons were conducted with Bonferroni's correction. ${ }^{*}=$ Differences are significant if $p<0.05$. Abbreviations: BYAACQ $=$ brief young adult alcohol consequences questionnaire, $\mathrm{AO}=$ alcohol only; $\mathrm{AMED}=$ alcohol mixed with energy drink.

Table 10. Energy drinks mixed with alcohol consumption and negative consequences.

\begin{tabular}{|c|c|c|c|c|c|c|c|c|}
\hline \multirow[b]{2}{*}{$\begin{array}{l}\text { BYAACQ } \\
\text { Group }\end{array}$} & \multicolumn{3}{|c|}{$\begin{array}{c}\text { Number of Energy Drinks on AMED } \\
\text { Occasion }\end{array}$} & \multirow{2}{*}{$\begin{array}{l}\text { Overall } \\
p \text {-Value }\end{array}$} & \multicolumn{3}{|c|}{ Pairwise Comparisons } & \multirow[b]{2}{*}{$\begin{array}{l}\text { Corrected } \\
p \text {-Value }\end{array}$} \\
\hline & $\begin{array}{l}\text { Low } \\
(0-4)\end{array}$ & $\begin{array}{c}\text { Moderate } \\
(5-8)\end{array}$ & $\begin{array}{l}\text { High } \\
(9-24)\end{array}$ & & $\begin{array}{l}\text { Low vs. } \\
\text { Moderate }\end{array}$ & $\begin{array}{l}\text { Moderate } \\
\text { vs. High }\end{array}$ & $\begin{array}{l}\text { Low vs. } \\
\text { High }\end{array}$ & \\
\hline \multicolumn{9}{|c|}{ Usual AMED Occasion } \\
\hline Men & $1.8(1.4)$ & $2.1(2.0)$ & $2.7(2.2)$ & $<0.0001 *$ & 0.782 & $<0.0001 *$ & $<0.0001 *$ & $<0.0001$ * \\
\hline Women & $1.5(1.0)$ & $2.1(1.6)$ & $2.0(1.4)$ & $<0.0001^{*}$ & $<0.0001 *$ & 1.000 & 0.001 * & $<0.0001$ * \\
\hline \multicolumn{9}{|c|}{ Past Month Heaviest AMED Occasion } \\
\hline Men & $2.5(2.1)$ & $3.3(3.1)$ & $3.7(2.8)$ & $<0.0001$ * & 0.004 * & 0.111 & $<0.0001 *$ & $0.002 *$ \\
\hline Women & $2.0(1.6)$ & $2.7(1.7)$ & $2.7(1.9)$ & $<0.0001 *$ & $<0.0001 *$ & 1.000 & $<0.0001 *$ & $0.005 *$ \\
\hline
\end{tabular}

Mean and SD (between brackets) number of energy drinks consumed on AMED occasions are shown. Post-hoc pairwise comparisons were conducted with Bonferroni's correction. ${ }^{*}=$ Differences are significant if $p<0.05 .{ }^{1}=p$-value corrected for the number of alcoholic drinks consumed. Abbreviations: BYAACQ = brief young adult alcohol consequences questionnaire; $\mathrm{AMED}=$ alcohol mixed with energy drink.

\section{Discussion}

The aim of the current study was to investigate energy drink and alcohol consumption in the context of risk-taking and experiences of alcohol-related negative consequences. Across the three countries studied, it was found that significantly less alcohol is consumed on AMED occasions compared with AO occasions (see Table 2). Consistent with this finding, significantly fewer negative alcohol-related consequences are reported for AMED occasions compared to AO occasions (see Table 4).

There are, however, differences between the countries in consumption levels, which have been discussed elsewhere [33]. For example in general the UK sample consumes more alcohol than the Dutch and Australian sample. However, the differences in alcohol consumption between $\mathrm{AO}$ and AMED occasions were consistent between countries and across risk-taking levels. Significantly less alcohol is consumed on AMED occasions than AO occasions. These findings are in line with previous research $[27,28]$ showing that AMED consumers in general drink more alcohol than $\mathrm{AO}$ consumers. However, using a within-subject design comparing AMED with AO occasions, decreased alcohol consumption on AMED occasions has been consistently found in studies around the world. 
The results suggests that low, medium or high levels of alcohol consumption depend on one's personality characteristics and are not related to the chosen non-alcoholic mixer.

The current analysis revealed that an increased level of risk-taking behavior was associated with increased alcohol intake and that energy drink consumption did not modify this. Specifically, this association was seen for both AMED and AO occasions and was more pronounced in the latter. This study adds to the literature that the level of risk-taking behavior was not clearly related to energy drink consumption. Across risk-taking levels, variation in the amount of energy drink consumed on AMED occasions was no more than a single $250 \mathrm{~mL}$ serving of energy drink for each country. When correcting for amount of alcohol consumed, there were no statistically significant differences in the number of energy drinks consumed on AMED occasions between the risk-taking groups. Thus, whereas the level of risk-taking behavior is unrelated to the amount of energy drink consumed on AMED occasions, the risk-taking level is associated with the overall amount of alcohol consumed, both on AO and AMED occasions. The data thus support our hypothesis that mixing alcohol with energy drinks has no relevant influence on alcohol consumption levels and subsequent negative alcohol-related consequences. Across countries it was consistently found that the relationship with risk-taking is driven by the amount of alcohol consumed and not by the amount of energy drinks consumed on AMED occasions. In contrast to the large differences observed for alcohol, the amount of energy drink consumed usually differed less than one $250 \mathrm{~mL}$ can between the risk-taking of BYAACQ levels. This was observed in both men and women.

Regarding reported alcohol-related negative consequences, there was also a strong association between the amount of alcohol consumed and the number of experienced past year negative alcohol-related consequences. With greater alcohol intake, either on AO or AMED occasions, more negative alcohol-related consequences were reported. The association between the number of energy drinks mixed with alcohol and negative alcoholrelated consequences were also statistically significant, but was less pronounced compared to the association with between alcohol intake and consequences. It is important to note that the observed difference in number of energy drinks consumed on AMED occasions between the lowest and highest BYAACQ groups was about one $250 \mathrm{~mL}$ serving of energy drink, which is much smaller than the corresponding increase of 3 to 4 alcoholic drinks (see Figure 1). Finally, as the chances of mixing energy drink with alcohol becomes larger when more alcohol is consumed, it is understandable that the total amount of alcohol consumed influences the relationship between energy drink intake and consequences.

The current findings are in line with previous research showing that higher levels of alcohol consumption are associated with increased risk-taking behavior and more frequently experiencing negative alcohol-related consequences [6-10]. The findings are also in agreement with previous meta-analyses showing that individuals who consume AMED do not consume more alcohol on AMED occasions compared to AO occasions [26-28]. Finally, the data confirm previous studies that conducted within-subject comparisons showing that, compared to AO occasions, consuming AMED does not increase negative alcohol-related consequences or risk-taking behavior [23,24].

The analysis revealed several sex differences. Men reported consuming significantly more alcohol than women, and more frequently. This was found for both AO and AMED occasions. In line, men reported significantly higher levels of risk-taking behavior compared to women and significantly more negative alcohol-related consequences. Among both men and women clear relationships between the level of risk-taking and alcohol consumption and between the level of experienced negative alcohol-related consequences and alcohol consumption were observed, whereas the differences in energy drink consumption were usually not significantly related to risk-taking levels or the number of reported negative alcohol-related consequences. Finally, in both men and women alcohol consumption on AMED occasions was significantly lower compared to the amount of alcohol consume on AO occasions. 
A strength of the current study is that the same survey was replicated in three countries. Validated questionnaires were used to assess risk-taking and negative alcohol-related consequences. The key research findings are consistent across the three countries, which strengthens the belief that the study outcomes can be generalized to other countries. While small differences in statistical significances were observed between the countries, the relationship between alcohol intake, risk-taking and negative consequences was evident in all three countries [33], whereas risk-taking was unrelated to the number of energy drinks consumed on AMED occasions.

A limitation of the current study is that the data were collected retrospectively. Therefore, recall bias may have influenced the study outcomes. Future prospective studies should therefore be conducted before these findings can be confirmed. Secondly, the samples under investigation comprised only students, in the age range of 18 to 30 years old. It is therefore unclear to what extend our findings can be generalized to other age groups and non-student populations. This is an important area for future research, as a recent comparison of student and non-student populations revealed that non-students consistently consume more alcohol and are involved in a greater number of negative alcohol-related consequences than students [31]. Third, in the surveys we assessed negative alcohol-related consequences. However, the items of the BYAACQ could be answered only with 'yes' or 'no', and therefore we were unable to assess the frequency of occurrence of these consequences. As there are significantly more AO occasions than AMED occasions, this may have an impact on the frequency of experiencing negative consequences. On the other hand, the impact of the latter may be relatively small, as a study that matched the frequency of AMED and AO occasions also reported lower odds of experiencing negative alcohol-related consequences on AMED occasions compared to AO occasions [24].

\section{Conclusions}

The data suggest that the level of risk-taking behavior is associated with the amount of alcohol consumed. In contrast, level of risk-taking behavior seems unrelated to the amount of energy drink consumed on AMED occasions. The experienced negative alcohol-related consequences associated with the drinking occasion are clearly related to the amount of alcohol consumed, whereas the amount of energy drink consumed had relatively limited impact. The data show that alcohol consumption levels among young adults are relatively high, and this has implications for both future research and policy makers. The current research suggest that they should focus on the negative effects of excessive consumption of alcohol per se. Focus on non-alcoholic mixers such as energy drinks does not help to limit overall alcohol consumption, nor does it reduce alcohol-related risk-taking or negative consequences.

Author Contributions: Conceptualization, J.C.V., C.A., A.S. data collection, J.C.V., S.B., S.J.J., data analysis, J.C.V., S.B., S.J.J., writing—original draft preparation, J.C.V.; writing-review and editing, J.C.V., S.B., S.J.J., C.A., A.S. All authors have read and agreed to the published version of the manuscript.

Funding: This research was funded by Red Bull GmbH, Utrecht University, Swinburne University, and the University of the West of England.

Institutional Review Board Statement: The studies were conducted according to the guidelines of the Declaration of Helsinki. The Dutch survey was reviewed by The Medical Ethical Review Board Twente, but no formal medical ethics approval was required, the UK survey was approved by the University of the West of England Faculty Ethics committee (approval number: HAS/14/03/57), and the Australian survey was approved by the Swinburne University Human Research Ethics Committee (Reference 2012/045).

Informed Consent Statement: Informed consent was obtained from all participants involved in the studies.

Data Availability Statement: The data is available on request from the corresponding author. 
Conflicts of Interest: Red Bull GmbH had no role in the design of the study; in the collection, analysis, or interpretation of data; in the writing of the manuscript, or in the decision to publish the results. Over the past 36 months, A.S. has held research grants from Abbott Nutrition, Arla Foods, Bayer, BioRevive, DuPont, Fonterra, Kemin Foods, Nestlé, Nutricia-Danone, and Verdure Sciences. He has acted as a consultant/expert advisor to Bayer, Coca-Cola, Danone, Naturex, Nestlé, Pfizer, Sanofi, Sen-Jam Pharmaceutical, and has received travel/hospitality/speaker fees from Bayer, Pfizer, Sanofi, and Verdure Sciences. C.A. has undertaken sponsored research, or provided consultancy, for a number of companies and organizations including Airbus Group Industries, Astra, British Aerospace / BAE Systems, UK Civil Aviation Authority, Duphar, Farm Italia, Carlo Erba, Ford Motor Company, ICI, Innovate UK, Janssen, LERS Synthélabo, Lilly, Lorex/Searle, UK Ministry of Defence, More Labs, Quest International, Red Bull GmbH, Rhone-Poulenc Rorer, Sanofi Aventis, Vital Beverages. Over the past 3 years, J.C.V. has received grants/research support from Janssen Research and Development, Danone, and Sequential Medicine, and has acted as a consultant/advisor for More Labs, Red Bull GmbH, Sen-Jam Pharmaceutical, Toast!, Tomo, and ZBiotics. S.B. has received funding from Red Bull GmbH, Kemin Foods, Sanofi Aventis, Phoenix Pharmaceutical, BioRevive, Australian Government Innovations Scheme and GlaxoSmithKline. S.J.J. has undertaken sponsored research for Pfizer, AstraZeneca, Merck, Gilead, Novartis, Roche, Red Bull GmbH, the Department for Transport, and Road Safety Trust.

\section{References}

1. Griswold, M.G.; Fullman, N.; Hawley, C.; Arian, N.; Zimsen, S.R.; Tymeson, H.D.; Venkateswaran, V.; Tapp, A.D.; Forouzanfar, M.H.; Salama, J.S.; et al. Alcohol use and burden for 195 countries and territories, 1990-2016: A systematic analysis for the Global Burden of Disease Study 2016. Lancet 2018, 392, 1015-1035. [CrossRef]

2. Rehm, J.; Gmel, G.E., Sr.; Gmel, G.; Hasan, O.S.; Imtiaz, S.; Popova, S.; Probst, C.; Roerecke, M.; Room, R.; Samokhvalov, A.V.; et al. The relationship between different dimensions of alcohol use and the burden of disease-An update. Addiction 2017, 112, 968-1001. [CrossRef]

3. Yuan, P.; Raz, N. Prefrontal cortex and executive functions in healthy adults: A meta-analysis of structural neu-roimaging studies. Neurosci. Biobehav. R. 2014, 42, 180-192. [CrossRef]

4. Kumar, S.; Porcu, P.; Werner, D.F.; Matthews, D.B.; Diaz-Granados, J.L.; Helfand, R.S.; Morrow, A.L. The role of GABA A receptors in the acute and chronic effects of ethanol: A decade of progress. Psychopharmacology 2009, 205, 529-564. [CrossRef] [PubMed]

5. Oscar-Berman, M.; Marinković, K. Alcohol: Effects on neurobehavioral functions and the brain. Neuropsychol. Rev. 2007, 17, 239-257. [CrossRef]

6. Berry, M.S.; Johnson, M.W. Does being drunk or high cause HIV sexual risk behavior? A systematic review of drug administration studies. Pharmacol. Biochem. Behav. 2018, 164, 125-138. [CrossRef] [PubMed]

7. Kovács, I.; Richman, M.J.; Janka, Z.; Maraz, A.; Andó, B. Decision making measured by the Iowa Gambling Task in al-cohol use disorder and gambling disorder: A systematic review and meta-analysis. Drug Alcohol Depend. 2017, 181, 152-161. [CrossRef]

8. Chen, S.; Yang, P.; Chen, T.; Su, H.; Jiang, H.; Zhao, M. Risky decision-making in individuals with substance use disorder: A meta-analysis and meta-regression review. Psychopharmacology 2020, 237, 1893-1908. [CrossRef]

9. Harmon, D.A.; Haas, A.L.; Peterkin, A. Experimental tasks of behavioral risk taking in alcohol administration studies: A systematic review. Addict. Behav. 2021, 113, 106678. [CrossRef]

10. Verster, J.C.; Kuerten, Y.; Olivier, B.; van Laar, M.W. The ACID-survey: Methodology and design of an online survey to access alcohol and recreational cocaine use and is consequences for traffic safety. Open Addict. J. 2010, 3, 24-31. [CrossRef]

11. Morris, D.H.; Treloar, H.R.; Niculete, M.E.; McCarthy, D.M. Perceived danger while intoxicated uniquely contributes to driving after drinking. Alcohol. Clin. Exp. Res. 2014, 38, 521-528. [CrossRef]

12. Sommers, M.S.; Lyons, M.S.; Fargo, J.D.; Sommers, B.D.; McDonald, C.C.; Shope, J.T.; Fleming, M.F. Emergency department-based brief intervention to reduce risky driving and hazardous/harmful drinking in young adults: A ran-domized controlled trial. Alcohol. Clin. Exp. Res. 2013, 37, 1753-1762. [CrossRef]

13. White, A.M. What happened? Alcohol, memory blackouts, and the brain. Alcohol Res. Health 2003, 27, 186. [PubMed]

14. Franzen, M.; Sadikaj, G.; Moskowitz, D.S.; Ostafin, B.D.; aan het Rot, M. Intra-and interindividual variability in the behavioral, affective, and perceptual effects of alcohol consumption in a social context. Alcohol. Clin. Exp. Res. 2018, 42, 952-961. [CrossRef]

15. Massa, A.A.; Subramani, O.S.; Eckhardt, C.I.; Parrott, D.J. Problematic alcohol use and acute intoxication predict anger-related attentional biases: A test of the alcohol myopia theory. Psychol. Addict. Behav. 2019, 33, 139. [CrossRef]

16. Rehm, J.; Shield, K.D.; Joharchi, N.; Shuper, P.A. Alcohol consumption and the intention to engage in unprotected sex: Systematic review and meta-analysis of experimental studies. Addiction 2012, 107, 51-59. [CrossRef] [PubMed]

17. Afshar, M.; Netzer, G.; Salisbury-Afshar, E.; Murthi, S.; Smith, G.S. Injured patients with very high blood alcohol concentrations. Injury 2016, 47, 83-88. [CrossRef] [PubMed]

18. Scalese, M.; Denoth, F.; Siciliano, V.; Bastiani, L.; Cotichini, R.; Cutilli, A.; Molinaro, S. Energy Drink and Alcohol mixed Energy Drink use among high school adolescents: Association with risk taking behavior, social characteristics. Addict. Behav. 2017, 72, 93-99. [CrossRef] [PubMed] 
19. Roemer, A.; Stockwell, T. Alcohol Mixed with Energy Drinks and Risk of Injury: A Systematic Review. J. Stud. Alcohol Drugs 2017, 78, 175-183. [CrossRef]

20. Woolsey, C.; Waigandt, A.; Beck, N.C. Athletes and energy drinks: Reported risk-taking and consequences from the combined use of alcohol and energy drinks. J. Appl. Sport Psychol. 2010, 22, 65-71. [CrossRef]

21. Berger, L.K.; Fendrich, M.; Chen, H.-Y.; Arria, A.M.; Cisler, R.A. Sociodemographic correlates of energy drink consumption with and without alcohol: Results of a community survey. Addict. Behav. 2011, 36, 516-519. [CrossRef] [PubMed]

22. Brache, K.; Stockwell, T. Drinking patterns and risk behaviors associated with combined alcohol and energy drink consumption in college drinkers. Addict. Behav. 2011, 36, 1133-1140. [CrossRef] [PubMed]

23. Peacock, A.; Bruno, R.; Martin, F.H.; Carr, A. The impact of alcohol and energy drink consumption on intoxication and risk-taking behaviour. Alcohol. Clin. Exp. Res. 2013, 37, 1234-1242. [CrossRef]

24. Peacock, A.; Bruno, R. Young adults who mix alcohol with energy drinks: Typology of risk-taking behavior. Addict. Behav. 2015, 45, 252-258. [CrossRef] [PubMed]

25. Peacock, A.; Droste, N.; Pennay, A.; Lubman, D.I.; Miller, P.; Newcombe, D.; Bruno, R. Self-reported risk-taking behavior during matched frequency sessions of alcohol versus combined alcohol and energy drinks consumption: Does co-ingestion increase risk-taking? Alcohol. Clin. Exp. Res. 2015, 39, 911-918. [CrossRef]

26. Verster, J.C.; Aufricht, C.; Alford, C. Energy drinks mixed with alcohol: Misconceptions, myths, and facts. Int. J. Gen. Med. 2012, 5, 187-198. [CrossRef]

27. Verster, J.C.; Benson, S.; Johnson, S.J.; Scholey, A.; Alford, C. Mixing alcohol with energy drink (AMED) and total alcohol consumption: A systematic review and meta-analysis. Hum. Psychopharmacol. 2016, 31, 2-10. [CrossRef] [PubMed]

28. Verster, J.C.; Benson, S.; Johnson, S.J.; Alford, C.; Godefroy, S.; Scholey, A. Alcohol Mixed with Energy Drink (AMED): A critical review and meta-analysis. Hum. Psychopharmacol. 2018, 33, e2650. [CrossRef] [PubMed]

29. de Haan, L.; de Haan, H.A.; van der Palen, J.; Olivier, B.; Verster, J.C. The effects of consuming alcohol mixed with energy drinks (AMED) versus consuming alcohol only on overall alcohol consumption and alcohol-related negative consequences. Int. J. Gen. Med. 2012, 5, 953-960.

30. Johnson, S.J.; Alford, C.; Stewart, K.; Verster, J.C. A UK student survey investigating the effects of consuming alcohol mixed with energy drinks on overall alcohol consumption and alcohol-related negative consequences. Prev. Med. Rep. 2016, 4, 496-501. [CrossRef]

31. Benson, S.; Verster, J.C.; Scholey, A. Consumption patterns of alcohol and alcohol mixed with energy drinks (AMED), in Australian students and non-students. Nutrients 2020, 12, 149. [CrossRef] [PubMed]

32. de Haan, L.; de Haan, H.; Olivier, B.; Verster, J.C. Alcohol mixed with energy drinks: Methodology and design of the Utrecht Student Survey. Int. J. Gen. Med. 2012, 5, 889-898. [PubMed]

33. Johnson, S.J.; Verster, J.C.; Benson, S.; Alford, C.; Scholey, A. A cross cultural comparison of negative alcohol-related consequences when mixing alcohol with energy drinks and other non-alcoholic beverages. Alcohol. Clin. Exp. Res. 2016, (Suppl. S1), 231A.

34. Sobell, L.C.; Agrawal, S.; Sobell, M.B.; Leo, G.I.; Young, L.J.; Cunningham, J.A.; Simco, E.R. Comparison of a quick drinking screen with the timeline followback for individuals with alcohol problems. J. Stud. Alcohol 2003, 64, 858-861. [CrossRef]

35. Roy, M.; Dum, M.; Sobell, L.C.; Sobell, M.B.; Simco, E.R.; Manor, H.; Palmerio, R. Comparison of the quick drinking screen and the alcohol timeline followback with outpatient alcohol abusers. Subst. Use Misuse 2008, 43, 2116-2123. [CrossRef] [PubMed]

36. de Haan, L.; Kuerten, Y.; Kuipers, E.; van Laar, M.W.; Olivier, B.; Verster, J.C. The RT-18: A new screening tool to assess young adult risk taking behavior. Int. J. Gen. Med. 2011, 4, 575-584.

37. Kahler, C.W.; Strong, D.R.; Read, J.P. Toward efficient and comprehensive measurement of the alcohol problems continuum in college students: The brief young adult alcohol consequences questionnaire. Alcohol. Clin. Exp. Res. 2005, 29, 1180-1189. [CrossRef]

38. Verster, J.C.; Herwijnen, J.; Olivier, B.; Kahler, C.W. Validation of the Dutch version of the Brief Young Adult Alcohol Consequences Questionnaire (B-YAACQ). Addict. Behav. 2009, 34, 411-414. [CrossRef] 